\title{
Tissue engineering today, not tomorrow
}

\author{
EuroSciCon Conference, November 17, 2006, The MI Centre, London, UK
}

\author{
Stephen M Richardson \\ Division of Regenerative Medicine, University of Manchester, Oxford Road, \\ Manchester, M13 9PT, UK \\ Tel.: +44 161275 5299; Fax: +44 161275 5289; E-mail: s.richardson@manchester.ac.uk
}

Tissue engineering has tremendous potential for the treatment of a wide range of medical conditions. In combination with this, the developments in the fields of embryonic stem cells (ES) and adult stem cells are leading to a whole new generation of therapies. H owever, for any treatment to be successful, a number of key issues must be addressed; in particular the choice of cell and scaffold types used and the methods required to overcome immune rejection of any transplant. The 1-day EuroSciC on meeting, at the MI Centre in London, UK, on N ovember 17, 2006, covered the recent advances in the fields of tissue engineering and regenerative medicine. Entitled 'Tissue Engineering Today, $\mathrm{N}$ ot Tomorrow', the meeting drew presenters from throughout Europe and as far afield as Japan. It focused on the key areas of development in stem cell differentiation, scaffold development and immune modulation, each with a clinical setting or scenario in mind.

The conference was opened by Dame Julia Polak (Imperial college, London, UK), who gave an interesting overview of the processes involved in developing tissue engineering from the laboratory into the clinic. Specific examples were given for the importance of biochemical and physical microenvironments on ES cell differentiation and how gene overexpression of osterix can induce bone formation. The use of bioglass for bone regeneration and collagen-polyglycolic acid composite scaffolds for bladder tissue engineering were also given. In conclusion, Dame Polak discussed how the fields of stem cells, tissue engineering, gene therapy, nanotechnology and regenerative medicine are all converging for clinical application to save lives. This gave a good introduction to the themes of the remaining talks.

\section{Application of novel}

methodologies for analysis of

cells, materials \& tissues

From cell isolation to analysis of engineered tissue constructs, analytical methods must be used that answer the questions asked and complement the systems used. To this end, a number of talks throughout the day described a range of novel methodologies that have been developed to aid mesenchymal stem cell (MSC) identification, analyze functional gene expression, assess load at a cellular level and analyze engineered tissues in real-time.

The first presentation of note was by Susan D onath (Miltenyi Biotec, Germany), who talked about the identification and isolation of stem cells from a variety of tissues. In particular, $D$ onath discussed the use of a range of CD markers to identify cells, including the use of CD 14, 34, 44, 45, 73, 105, 133, 166 and 271. Examples were given of how positive and negative screening for combinations of $C D$ markers could define the phenotype, such as MSCs that are negative for CD 14, 34, 45, 133 and positive for CD 44, 73, 90, 105 and 166. 0 wing to the fact that CD271+ cells had increased proliferation rates over plastic adherent, unsorted M SC S, CD 271 was suggested as the best marker for isolating M SCs from bone marrow. Other isolation methods were introduced for a number of applications, including CD 56+ isolation of muscle progenitor cells for muscle regeneration in deuchenne muscular dystrophy and CD 133' isolation of blood-derived stem cells for myogenic differentiation.

Farzin Farzaneh (King's College, London, UK) talked on functional analysis of the genome for the identification of novel stem cell regulatory factors. The method used retroviral insertional mutagenesis to insert a gene that may cause differentiation, apoptosis or other selectable phenotypes, which allows a clonal population with the desired phenotype to be isolated and the effect of the gene to be examined. Recent modifications have been made to improve the efficiency of the process, in which biotinylated retroviruses or lentiviruses are paramagnetically labelled to concentrate the vectors. Targeting ligands can also be added using the biotin label and a 125-fold concentration has been shown to cause a 4200 -fold increase in infective titer of the virus.

In another talk, Catherine Sarraf (Westminster University, London, UK) described two methods of measuring force at a cellular level, the culture force monitor (CFM), which calibrates the contractile profiles of tissues and tensioning-CFM, which applies and records load. When force was applied to resting dermal fibroblasts they elongated and aligned with the direction of force, and transmission electron microscopy (TEM) showed changes in morphology and nuclear arrangement. $D$ ata were also presented for the effects on the production of collagen by porcine aortic wall cells and the ability of load to evoke a myofibroblast-type response from M SC s. There was also evidence for the similarities between mechanical 
load and medium composition for the induction of various cells types from stem cells.

Andre $\mathrm{N}$ eves (University of Cambridge, Cambridge, UK) talked on the development of scaffold screening methods to assess engineered meniscal cartilage using both M RI and magnetic resonance spectroscopy (MRS) technologies. Current treatments of mensical defects are unsuccessful and can lead to arthritic problems, while synthetic prosthetics and allogeneic transplants have limited success. $\mathrm{N}$ ovel tissue engineering strategies using cell-seeded polyethylene terephtalate (PET) constructs are being developed, however evaluations of constructs are invasive and provide slow feedback. MRI and $M R S$ are noninvasive and give rapid or even real-time feedback. They used 31P-MRS to study cell energetics and viability and M RI to examine parameters within bioreactors, such as flow rate, perfusion, porosity and cell density and distribution. An example was given in which the geometry of PET scaffolds was changed from random to evenly spaced fibers. Results showed that scaffold geometry affected flow rate and cell viability. This information was compared with histological data, which showed no cells in the center of the random-fiber scaffold, but even cell distribution in the oriented scaffold.

\section{Development of new}

generations of scaffolds for

tissue engineering applications

The choice of biomaterial is one of the biggest determinants in a successful tissue engineering approach. Scaffold architecture must be controlled carefully to ensure optimal porosity and pore interconnectivity, while being mechanically stable enough to protect cells from factors, such as load. In addition, new generations of scaffolds are being developed to incorporate the ability to release factors, such as growth factors, to aid cell proliferation, differentiation or matrix formation, in a controlled manner. An overview of the development of this new generation of biomaterials was given by Nureddin
Ashammakhi (Keele University, Staffordshire, UK), who provided examples of the first generation of bioinert but resorbable biomaterials, such as bone screws, in which the space left as the screw degrades is replaced with tissue; however, in many cases, this tissue is fibrous, not bone. As a result, the second generation of biomaterials included bioactive molecules to aid tissue regeneration, for example, titanium screws with titanium oxide on the surface. Biactive glasses, such as Perioglas ${ }^{\circledR}$, were developed with a silicone oxide-rich layer and a calcium phosphate layer; however, since glass is very brittle, these materials were integrated with other biomaterials, such as polycaprolactone (PCL). M ore recently, the third generation of multifunctional biomaterials have been developed. These materials combine the benefits of the second generation biomaterials, but have active factors or antibiotics to aid tissue regeneration or reduce the chance of infection around the implant site. Ashammakhi's group have looked at local antibiotic and anti-inflammatory release profiles from biomaterials and demonstrated that these compounds do now affect tissue formation. The addition of bisphosphonates to biomaterials for bone regeneration in patients with osteoporosis and the effect of biomaterial choice and fiber orientation on tissue formation were al so discussed in relation to development of the generation of new biomaterials.

T M orton (Ludwig Boltzman Institute for Experimental and Clinical Traumology, Vienna, Austria) talked about the use of electrospinning to create a biomimicking nanofiber matrix of fibrin or fibrinogen. The method used a protein solution, which is passed through an electric field to form a jet that is deposited on either a static or rotating collector screen. Fibrin and fibrinogen give matrices that differ in porosity, fiber thickness and density and the electrical charge on the pump gave differences in fiber thickness. Fibers could be formed between $100 \mathrm{~nm}$ and $3 \mu \mathrm{m}$ and showed similarities with native tissue fibers. H uman fat-derived
M SC s cultured within the matrices persisted over 14 days and the addition of either fibroblast growth factor (FGF)-2 or bone morphogenetic proteins (BM Ps) altered cell phenotype and cell orientation within the matrix.

$\mathrm{H}$ ossein Hosseinkhani ( $\mathrm{N}$ ational Institute for M aterials Sciences, Japan) described a new inducible angiogenesis carrier for cardiomyocyte transplantation. The work used a polyamphilic nanofiber (PAN) matrix, which was combined with growth factors, such as transforming growth factor (TGF)- $\beta$ and FGF-2. Investigations in nude mice demonstrated that the combined PAN/FG F-2 matrix caused neovascularization which neither the PAN matrix nor FG F-2 could do alone. When ${ }^{125}$ FGF-2 was used without the PAN matrix it was lost within 7 days of implantation, however, when the 125I-FGF-2 was implanted within the PAN matrix, $50 \%$ of the PAN matrix had been degraded by day 30 , as had $90 \%$ of the FGF-2. Further tests using cardiomyocytes revealed that a combination of cells, FGF-2 and PAN matrix was required for the production of a new tissue and survival of the cells and that the lack of either cells, growth factor or PAN matrix caused the system to fail. When cells were implanted alone there was no cell survival after 4 weeks. The results suggested that implantation of cardiomyocytes and FGF-2 within a PAN matrix could be used to treat myocardial infarction and proved how important a suitable scaffold is in successful tissue engineering strategies.

Kevin Shakesheff (University of N ottingham, N ottingham, UK) introduced a novel hydrogel, which is a combination of high-melting temperature porous Polyol-lactic acid (PD LLA) particles and a low-melting temperature polylactic-co-glycolic acid (PLGA)/polyethylene glycol (PEG) copolymer as a binding component. This hydrogel is a free flowing liquid at room temperature, however, at $37^{\circ} \mathrm{C}$, the PLGA/PEG undergoes a glass transition and becomes a solid, thus binding the PDLLA. By altering the proportion of the two components, the 
gel properties and glass transition temperature can be altered. They are currently developing this method for the treatment of osteochondral defects and hope to have a clinically viable treatment in approximately 3 years.

The final talk on the issue of biomaterials was given by Julian Chaudhuri (University of Bath, UK), who presented work on the development of scaffolds and bioreactors for clinical-scale tissue engineering. In light of the facts that static cultures lead to gradients of factors, such as nutrients and $\mathrm{pH}$, and current industrial bioreactors are not suitable for culturing 3D constructs, systems must be developed to ensure that the chosen scaffold is cultured in a manor that provides adequate nutrients to the cells embedded within it. W ithin a scaffolds, the highest concentration is found within $100-200 \mu \mathrm{m}$ of a pore. This also represents the maximum distance of a cell from a capillary in vascularized tissues and, within scaffolds, when cells are over $200 \mu \mathrm{m}$ from a nutrient supply, there is evidence of necrosis. Chaudhuri presented work on a phase inversion spin-casting method for producing hollow-fiber PLGA scaffolds. The structure of these scaffolds is altered, depending on whether PDLLGA or $P_{L} L G A$ is used and these changes affect cell attachment and proliferation. Chaudhuri also presented work in which ATDC-5 cells and STRO-1+ stem cells were cultured in al ginate/chitosan beads in the presence of TGF- $\beta_{3^{-}}$ containing medium. Following 23 days culture in either static or perfused bioreactor culture, differences were noted in cell distribution, chondrogenesis and presence of lacunae, which suggests that bioreactor culture affects cell phenotype.

Overcoming immune rejection by harnessing the potential of stem cells

A number of presentations throughout the day covered the issue of immune rejection of transplanted cells and tissues. W hile developments in cell differentiation methods and scaffold evolution offer the prospect of clinically viable treatments, allogeneic cell transplants will still have issues with rejection unless a suitable method of immune modulation is developed or a suitable population of immune privileged cells are found. A number of talks throughout the day covered this area of research. The first was by Paul Fairchild (University of Oxford, UK), who talked about ES cells, while later talks by S Wolbank (Red Cross Transfusion Centre for Upper Austria, Austria), Xiao N ong-Wang (University of $\mathrm{N}$ ewcastle, UK) and M arta C alatayud (Vall d'H ebron H ospital, Barcelona, Spain) concentrated on adult stem cells.

Fairchild presented data from experiments in mice that demonstrated that ES cells are not immune privileged allogeneically, even though they have low MHC class I and no MHC class II. Despite this fact, transplantation of allogeneic male ES cell-derived tissues results in an immune response, suggesting other factors are involved. Similarly, in a mouse model where skin from a male mouse was engrafted onto a female mouse, the tissue was rejected. $\mathrm{H}$ owever, when ES cells were differentiated into immature dendritic cells and engrafted with the male skin, the graft was accepted. D endritic cells are cells in the immune system that dictate immune response versus immune tolerance, although interestingly, only immature not mature ES cell-derived dendritic cells blocked the immune response. The timing of the addition of dendritic cells was also found to be important. Cells engrafted approximately 1 month before skin grafting inhibited the immune reaction, but when cells were implanted 2 months prior, the cells died and when cells were implanted only 2 days before the engraftment, the graft was rejected. In conclusion, Fairchild stated that human ES cells should be capable of differentiating into cells that could allow immune tolerance for ES cell-derived tissues for regenerative medicine.

Xiao N ong-Wang gave a talk on graftversus-host disease (GVHD), again using skin grafts as a model system. This work used adult MSCs and umbilical cord blood stem cells (UCBSC) rather than ES cells. When M SC s or U CBSC S were added at the $T$-cell activation stage, there was some decrease in skin damage and GVHD; however when stem cells were added after $\mathrm{T}$-cell activation, there was no decrease in tissue damage. A significant decrease in tissue damage was observed when stem cells were preincubated with interferon (IFN)- $\gamma$ or TN F- $\alpha$ and then implanted at the T-cell activation stage. A high ratio of stem cells to responder cells was required to see an effect, with minimum ratios being between 5:1 and 10:1. In conclusion, it was stated that M SC $s$ and U CBSC $s$ are not immunogenic, but are immunosuppressive and may have potential for use in treating GVH D.

Wolbank compared the immunomodulatory effects of adult M SC $s$ from adipose tissue (AM SC), adult stem cells from amniotic (H AM C) and epithelial cells from amniotic membrane (HAEC). Transplantation studies showed no immune reaction, suggesting immunomodulation by all three cell types. When allogeneic AM SC, HAMC or HAEC cells were co-cultured with phytohemaglutinin-activated peripheral blood mononuclear cells (PBMCS), the cells suppressed PBMC activation and an increase in stem cell number decreased PBMC proliferation. This effect was shown to be cell contact-dependent as co-cultures without contact showed no inhibition, regardless of M SC cell number. Increasing cell passage (up to six passages) had no effect on immunomodulatory effect; however cryopreservation did decrease the inhibitory effect. The immunomodulatory ability of M SC s was also not affected by preincubation with either IFN $-\gamma$ or T N F- $\alpha$.

In the last of the talks on immunomodulation, $C$ alatayud presented work from a clinical trial that attempted to overcome immune rejection of ocular surface implants by using limbal stem cells grown on amniotic membranes. Limbal deficiency, in which the conjunctival epithelium covers the cornea, leads to functional blindness, pain and a bloodshot, red eye. While there are many conditions that can cause limbal 
deficiency, limbal allografting can only be achieved with immunosuppression. $\mathrm{H}$ owever, data were presented from a clinical trial in which limbal stem cells were expanded ex vivo on amniotic membranes and then engrafted without immunosuppression. A total of 15 cases were treated in 12 patients, all with limbal stem cell disease, of which ten cases were autotransplants from a healthy eye, four were allogeneic grafts from family members and one was a cadaveric graft. $0 \mathrm{f}$ the 15 treatments, which had an average follow-up of 15 months, there was ocular surface restoration in $80 \%$, and only two cases failed due to complications. Calatayud explained that amniotic membrane as a substrate to grow limbal stem cells was the ideal choice since it favors the expansion of corneal epithelium and the microenvironment of the ocular surface is the key factor in the stability of a new epithelium.

\section{Prospects for the future}

In conclusion to his talk, Farzaneh compared the synergy between descriptive (e.g., mRNA or protein expression microarrays) and functional analysis with the synergy between a street map and a list of street names, in that one alone will not necessarily give you the all of the information you require. That concept could also be applied to the synergies between the types of cell used for tissue engineering and the types of scaffolds used. A succesfful approach must incorporate the best qualities of the two strands and equal care must be given to decisions on both aspects.
The work presented by $\mathrm{C}$ alatayud demonstrates that the clinical application is possible today, not tomorrow, and in many of the other talks, the presenters demonstrated a drive to clinic that will surely mean a successful application in the very near future. This enthusiasm must be tempered by a recognition of the broader aspects of the work, regulatory and ethical, especially where ES cells are concerned. H owever, the potential of these cells if the technical issues can be overcome are seemingly limitless and, with the correct associations of academic scientists, industrial scientists and clinicians, tissue engineering and regenerative medicine have the ability to cure countless medical conditions and improve the lives of billions of people worldwide. 